\title{
二十面体准晶对非晶形成影响的模拟*
}

\author{
周国荣吴佑实张川江赵芳 \\ (山东大学 (南校区) 材料科学与工程学院, 济南 250061)
}

\begin{abstract}
摘要 采用分子动力学模拟技术, 以液态金属 $\mathrm{Ni}$ 为例, 研究了在不同冷却条件下形成晶体及非晶的过程. 模拟 采用镶嵌原子法 (EAM) 作用势, 得到了不同温度、不同冷却速度下 $\mathrm{Ni}$ 的径向分布函数以及原子组态变化的重要 信息,利用键对分析技术探讨了二十面体准晶对非晶形成的影响 .
\end{abstract}

关键词: 二十面体准晶, 分子动力学模拟, 镶嵌原子法作用势

中图分类号： O753.3, O641

近年来, 计算机模拟技术已经发展成研究液态 结构信息的重要手段, 已取得了许多重要进展 ${ }^{[1-3]}$, 研究方法也在不断完善 ${ }^{[4-6]}$. 对于非晶形成的原因 目前还未有定论, 张川江等人 ${ }^{[7]}$ 认为二十面体准晶 对非晶的形成有重要的影响. 本文采用 Johnson 等 人的镶嵌原子方法 ${ }^{[8]}$ (embeded atom method, EAM), 用分子动力学模拟方法研究了金属 $\mathrm{Ni}$ 的液 固转变动力学及热力学过程, 探讨了二十面体准晶 对非晶形成的影响.

\section{1 原子间相互作用势}

根据 EAM 模型, $N$ 个原子体系的总能量可表 示成偶势与镶嵌原子能量之和

$$
E_{\mathrm{tot}}=\sum_{i} F\left(\rho_{i}\right)+\frac{1}{2} \sum_{i} \varphi(r) ; \rho_{i}=\sum_{j \neq i} f(r)
$$

其中, $\rho_{i}$ 是 $i$ 处的电子密度, $f(r)$ 为 $i$ 原子周围第 $j$ 个原子贡献的电子密度, $r$ 为原子间距离, $F\left(\rho_{i}\right)$ 为 原子 $i$ 的镶嵌能; $\varphi(r)$ 为传统的偶势. 关于镶嵌 能、电子密度和两体势的确定方法及势函数中参数 的选择见文献 ${ }^{[9]}$.

\section{2 分子动力学模拟的基本过程}

采用分子动力学模拟方法, 对象是施加周期性 边界条件的立方盒子中的 500 个原子, 模拟的时间 步长为 $3.5 \mathrm{fs}$, 小于原子的振动周期. 首先, 体系在 $2000 \mathrm{~K}$ 下弛豫 20000 个时间步长, 使其达到平衡 态. 然后, 分别以 $k_{1}=4 \times 10^{13} \mathrm{~K} \cdot \mathrm{s}^{-1}, k_{2}=4 \times 10^{11}$
$\mathrm{K} \cdot \mathrm{s}^{-1}$ 的速率进行冷却, 每隔 $200 \mathrm{~K}$ 记录体系的构 型, 得到径向分布函数; 动力学参数和热力学参数 每隔 $10 \mathrm{~K}$ 记录一次.

\section{3 结构分析方法}

\section{1 径向分布函数 ${ }^{[10]}$}

径向分布函数 $g(r)$ 与结构衍射实验得到的干 涉函数互为 Fourier 变换, 是理论与实验对照的基本 依据, 也是描述液态和非晶态等无序体系的基本方 法.

\section{2 原子的平均能量}

体系的内能由动能和势能两部分组成. 根据 原子间相互作用的势能函数, 计算出作用在每个原 子上的力, 通过求解牛顿运动方程得到每个原子的 速度及动能. 原子的平均能量与原胞的体积变化 类似, 也是温度的函数, 因此也可以通过内能的变 化来了解体系结构的转变.

\section{3 均方位移}

均方位移 (mean square displacement, MSD) 定 义为

$$
r^{2}(t)=\frac{1}{N}\left\langle\sum_{i=1}^{N}\left|r_{i}(t)-\left[r_{i}(0)\right]\right|^{2}\right\rangle
$$

$r_{i}(0)$ 为原子 $i$ 在 0 时刻的位移, $r_{i}(t)$ 为原子 $i$ 在 $t$ 时刻的位移. 均方位移随时间的变化可用来表征 液态金属原子的扩散行为.

\section{4 对分析技术}

对分析技术 (pair analysis technique, PA) 能提供 

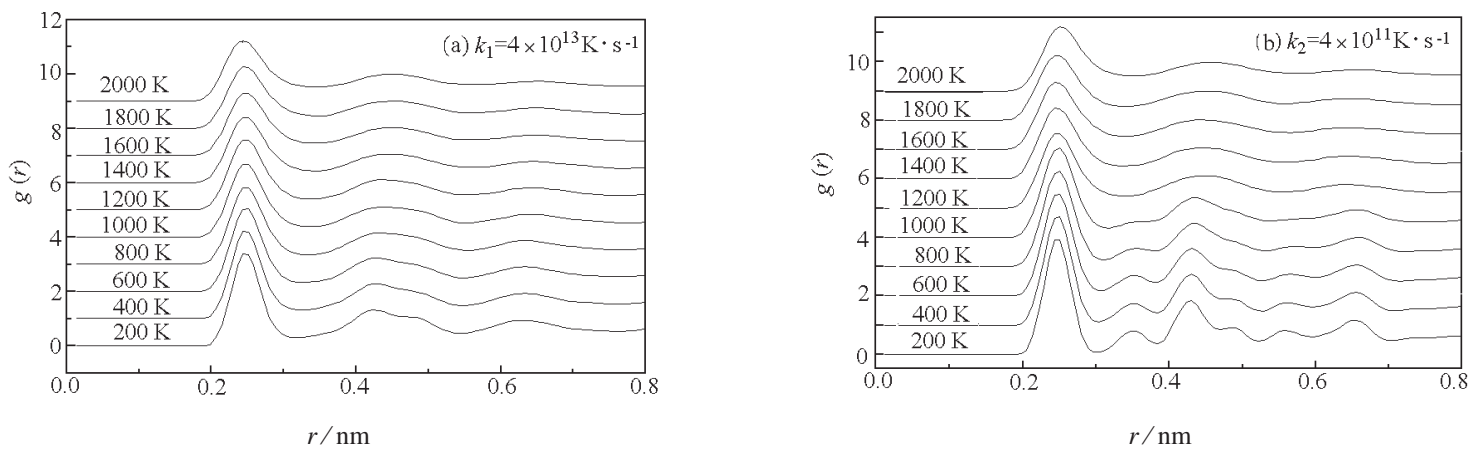

图 1 冷却速率分别为 $k_{1}(\mathrm{a})$ 和 $k_{2}(\mathrm{~b})$ 时不同温度下 $\mathrm{Ni}$ 的径向分布函数

Fig. 1 Pair correlation function of Ni at different temperatures by a cooling rate of $k_{1}(\mathrm{a})$ and $k_{2}(\mathrm{~b})$

研究液态金属结构演变规律的重要参数. 所谓的对 分析技术是指用两个原子及其与周围共有原子之间 的成键关系来描述这对原子. 当两个原子之间的距 离小于或等于径向分布函数第一谷所确定的最邻近 距离, 则称这两个原子成键. 对分析技术采用四个 指数 ( $i j k l)$ 来表征原子对的成键关系. $k=1$ 表示两 个原子构成一个键对, $j$ 表示与键对的两个原子都 构成近邻的原子数, $k$ 表示这些近邻原子之间也成 键的数目, $l$ 是标定在其它三个数字相同的情况下 组态还有差别的情况.

几种常见的 $\mathrm{H}-\mathrm{A}$ 键对指数所代表的结构模式 与典型的晶体结构的关系如下: 1551 和 1541 键对 表征二十面体结构, 1431 键对表征一定程度的无序 性。这三种键对在典型液态或非晶态中都会大量存 在; $f c c$ 型晶体结构以 1421 键对为特征键对; 1422 键对表征 $h c p$ 型晶体结构; 表征 $b c c$ 型晶体结构的 特征键对是 1661 和 1441 键对. 体系中各种多面体 可由特定的键对种类和数目来构成, 例如 12 个 1551 键对可以构成以一个原子为中心, 周围有 12 个原子的二十面体结构, 两个二十面体共用一个公 用面则可构成双二十面体。

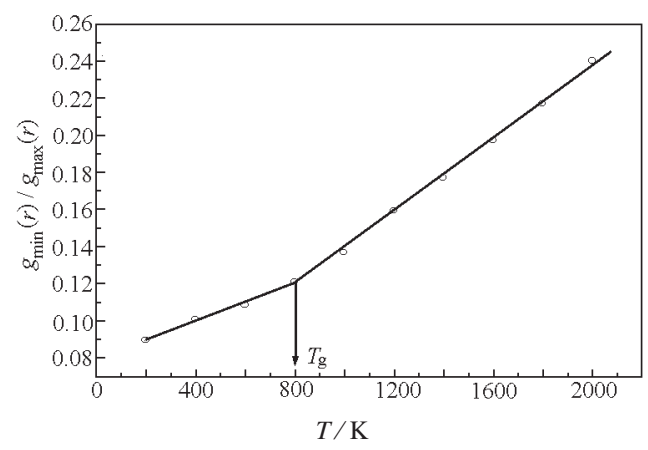

图 2 冷却速率为 $k_{1}$ 时的 $g_{\text {min }}(r) / g_{\text {max }}(r)$ 随 温度的变化

Fig. $2 g_{\min }(r) / g_{\max }(r) v s T$ at cooling rate of $k_{1}$

\section{4 结果和讨论}

\section{1 径向分布函数与有序性分析}

由图 1 (a) 可以看出, 在温度从 $2000 \sim 200 \mathrm{~K}$ 的 变化过程中, 径向分布函数的第一峰逐渐升高, 表明 每个原子第一近邻原子的数目越来越多, 相邻原子 成键的几率越来越大, 说明体系的无序度不断下降, 短程有序度不断上升. 在从 $800 \sim 200 \mathrm{~K}$ 降温过程 中, 径向分布函数的第二峰逐渐发生䢃裂。这是非 晶形成的特征. 由图 2 所示, 在 $k_{1}=4 \times 10^{13} \mathrm{~K} \cdot \mathrm{s}^{-1}$ 下, 非晶转变温度为 $800 \mathrm{~K}$.

同图 1 (a) 相比, 图 1(b)中 $2000 \sim 200 \mathrm{~K}$ 变化 过程中, 径向分布函数的第一峰变化更大. 表明, 在 该冷却速率下, 随温度的降低, 体系的有序度进一步 加强. 到 $1000 \mathrm{~K}$ 时, 曲线第一峰与第二峰之间出 现小峰, 这时有序度已达到一定的程度, 可能标志着 晶体的产生.

\section{2 相变热力学}

图 3 给出了冷却过程中能量随温度的变化. 由 图可见, 在 $k_{1}$ 条件下, 能量的变化是连续的, 对应非 晶的形成; 而 $k_{2}$ 下的能量曲线在 $1050 \mathrm{~K}$ 左右陡

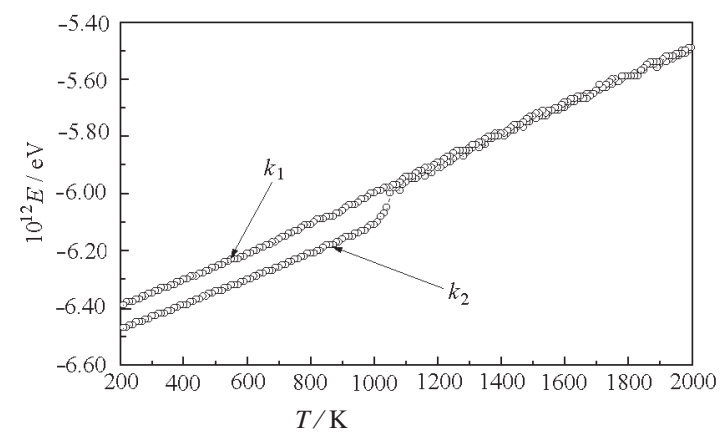

图 3 在不同冷却速率下总能量随温度的变化

Fig. 3 The total energy vs quenching temperature at cooling rate of $k_{1}$ and $k_{2}$ 


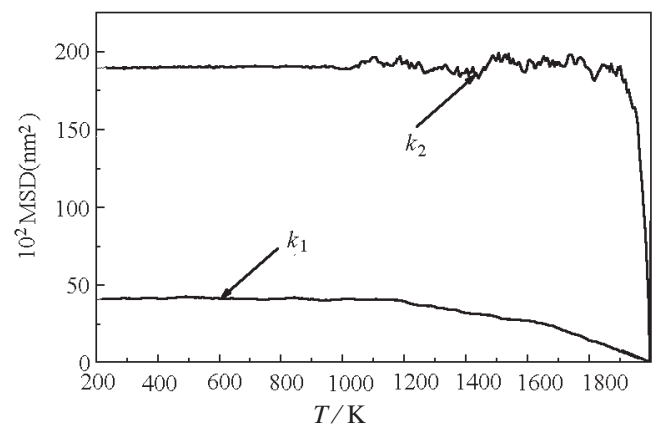

图 4 均方位移 (MSD) 随温度的变化关系

Fig. 4 Mean square displacement (MSD) vs temperature

降, 这表明结晶已发生。该结晶温度低于纯 $\mathrm{Ni}$ 结晶 的实验温度, 这是由于分子动力学模拟凝固过程容 易导致过冷, 且冷却速率越快, 过冷度越大.

\section{3 相变动力学}

由图 4 可见, 凝固过程中原子位置的重排对冷 却速率十分敏感. $k_{1} 、 k_{2}$ 的冷却速率相差不大, 而 MSD 相差较大。原子位移在液态和过冷液态区逐 渐增大, 说明结晶是原子作大范围的扩散重新定位 的过程. 而 MSD 在非晶形成过程中变化很小, 也 就是说原子不再作大范围的迁移, 只在平衡位置附 近振动. 这在凝固产物中表现得更加明显, 这是由 于其冷却速率过快, 使原子来不及扩散形成的.

\section{4 键对分析}

图 5 给出了在冷速为 $k_{1}=4 \times 10^{13} \mathrm{~K} \cdot \mathrm{s}^{-1}, k_{2}=$ $4 \times 10^{11} \mathrm{~K} \cdot \mathrm{s}^{-1}$ 条件下液态金属 $\mathrm{Ni}$ 的七种键对相对 数目随温度的变化关系. 如图 5(a)所示, 在 $k_{1}$ 冷 速下, 表征二十面体结构的 1551 和 1541 键对的数 量随温度下降呈连续上升趋势, 1551 和 1541 键对 在熔态时共占成键总数的 $20 \%$ 左右, 随温度的下 降, 其数量明显增加, 其中 1551 键对增加更快, 两者 最终共占成键总数的 $38 \%$ 左右, 说明由二十面体结

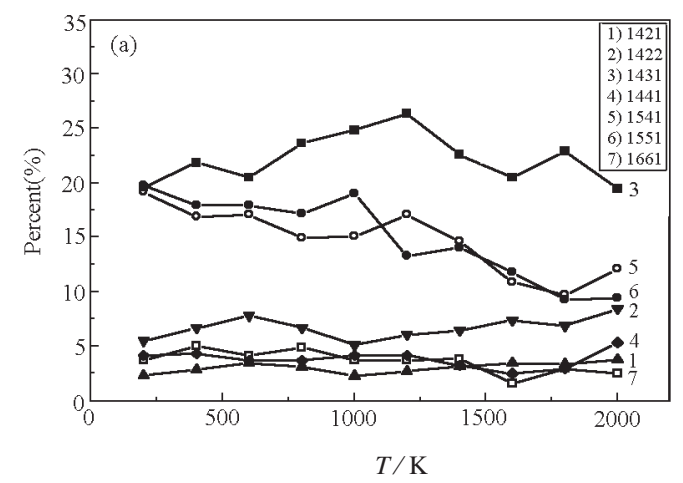

构单元组成的原子团簇在该系统中起重要作用. 即 在快冷条件下, 液态 $\mathrm{Ni}$ 将形成非晶态结构, 与 $g(r)$ 曲线上第二峰的䢃裂一致. 由图还可看出, 1431 键 对一直在体系中占主要地位, 并且随温度变化不大; 1551、1541 和 1431 键对占液态键对总数的 $50 \%$ 左 右, 占有绝对优势. 它们是典型液态中经常出现的 键对, 说明液态中大量存在的 1551、1541 和 1431 键 对在过冷条件下从高温熔体传递到非晶体中, 体现 了高温熔体结构与非晶态结构的相关性. 而如图 5 (b) 所示, 在 $k_{2}$ 冷速下, 这三种键对数量总体呈下降 趋势. 其中, 1551 键对在温度降到 $200 \mathrm{~K}$ 时几乎完 全消失, 1541 键对也仅占 $7 \%$ 左右. 这说明在较慢 的冷却速率条件下, 1551、1541 和 1431 键对可以有 足够的时间进行分解转化, 形成有利于稳定存在的 键对.

表征 $f c c$ 型晶体结构和表征 $h c p$ 型结构的 1421 键对和 1422 键对所形成的小团簇数量随温度 变化与前几种键对的情形有较大的差别. 图 5(a) 表明, 在 $k_{1}$ 冷速下, 它们的相对数目在整个冷却过 程中基本不变, 说明在较快冷速下液态 $\mathrm{Ni}$ 中的 $f c c$ 型晶体结构和 $h c p$ 型晶体结构来不及转化就保留 到非晶中. 而在 $k_{2}$ 冷速下 (图 5(b)), 当温度降至 $1200 \mathrm{~K}$ 以后, 它们的相对数目急剧增加, 其总量由 冷却前的约 5\% 增加至 50\% 左右, 说明体系中 $f c c$ 型晶体结构和 $h c p$ 型晶体结构单元占主导地位.

在图 5(a)中 ( $k_{1}$ 冷速下), 表征 $b c c$ 型晶体结构 的 1661 和 1441 键对所形成的小团簇的数量在整 个冷却过程中总体略显上升趋势, 最终约占总量的 $7 \%$. 而在 $k_{2}$ 冷速下, 1661 和 1441 键对数量由 2 $000 \mathrm{~K}$ 冷却到 $1050 \mathrm{~K}$ 的过程中呈明显上升趋势, 此 后迅速减少, 到 $200 \mathrm{~K}$ 时两者仅占总量的 $4 \%$. 这说 明在冷速较小的情况下, 在 $\mathrm{Ni}$ 的结晶过程中伴随着 1661 和 1441 键对向 1421 键对和 1422 键对的转

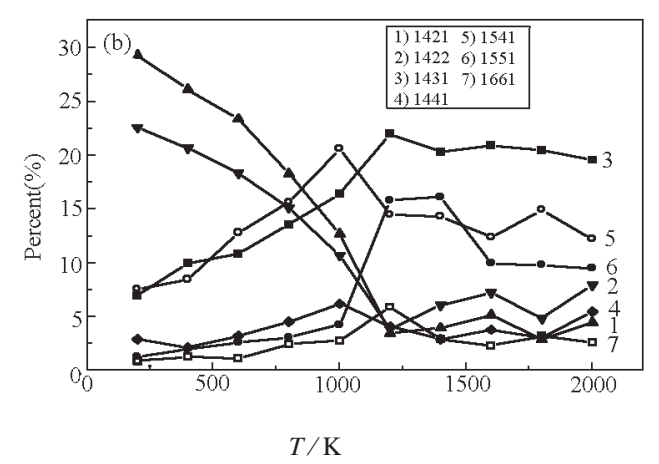

图 5 冷却速率分别为 $k_{1}(\mathrm{a})$ 和 $k_{2}(\mathrm{~b})$ 时不同温度下 $\mathrm{Ni}$ 的键对比例

Fig. 5 The ratio of bonded pair of Ni at different temperature by the cooling rate of $k_{1}(\mathrm{a})$ and $k_{2}(\mathrm{~b})$ 
换, 即由 $b c c$ 型晶体结构向 $f c c$ 型晶体结构和 $h c p$ 型晶体结构的转化。

\section{5 结 论}

(1)在较慢冷速下, 在 $\mathrm{Ni}$ 的结晶过程中会出现 局域团簇结构转化的现象. 表征 $b c c$ 型晶体结构 的 1661 和 1441 键对在结晶过程中出现向 1421 键 对和 1422 键对结构的转化, 最终形成 $f c c$ 型晶体结 构和 $h c p$ 型晶体结构占优的晶体.

（2）在较快冷速下, 表征二十面体结构的 1551 和 1541 键对的数量在整个温区变化最大.从统计 热力学的观点来看, 系统的结构因子 $S(q)$ 和径向 分布函数 $g(r)$ 的形状应由所有参与成键的键对结 构数的统计平均值确定, 而其形状应主要由变化程 度较大的键对数决定. 由此可见, 二十面体结构的 存在是造成 $g(r)$ 曲线出现第二峰䢃裂的原因。因 此, 可以认为二十面体准晶就是非晶形成的主要原 因. 在液态中存在大量由二十面体结构单元组成 的原子团簇, 其体积相对于其他结构团簇的体积要 大得多, 在冷速较快的条件下, 它们会在熔体中产 生巨大的“尺寸效应”, 阻碍了其他结构团簇向稳定
结构的转化. 另一方面, 它们本身也来不及向其他 稳定结构的转化就已经被“冻结”到非晶中。而且 在快速冷却过程中, 体系也没有时间向它们提供足 够的能量来克服自身的势垒, 从而导致整个体系的 大部分结构由液态保留到了固态, 从而迅速形成了 非晶。

\section{References}

1 Fincham, D. ; Heyes, D. M. Adv. Chem. Phys., 1985, 63: 493

2 Allen, M. P. ; Tildesley, D. J. Computer simulation of liquid. Oxford: Clarendon Press, 1987

3 Brandt, E. H. J. Phys. : Condens. Matter, 1989, 1: 9985

Li, D. H. ; Li, X. R. ; Wang, S. J. Phys. F, 1986, 18: 309

5 Honeycutt, J. D. ; Andersen, H. C. J. Phys. Chem., 1983, 91 : 4950

6 Swope, W. C. ; Andersen, H. C. Phys. Rev., 1990, B41: 7024

7 Zhang, C. J. ; Wu, Y. S. ; Cai, X. L. ; Zhou, G. R. J. Phys. :

Condens. Matter, 2001, 13: 647

8 Johnson, R. A. Phys. Rev., 1988, B37: 3927

9 Chen, S. P. ; Voter, A. F. ; Boring, A. M. J. Matter. Res., 1990, 5(5): 955

10 Waseda, Y. The structure of non-crystalline materials. New York: MCGRAM-Hill, 1981: 292

\title{
Molecular Dynamics Simulations of the Effect of Icosahedral Quasicrystal on the Formation of Amorphous*
}

Zhou Guo-Rong Wh Yhang Chuan-Jiang Z Zhang

( College of Materials Science and Engineering, Shandong University ( Southern Campus), Jinan 250061)

\begin{abstract}
By means of molecular dynamics simulation technique, the solidification process of Ni under different cooling rates has been studied. The variation of potential energy with temperature and the relationship between the energy and the structure of molecular-cluster have also been studied. With pair distribution function and pairs analysis method, the local symmetry of molecular-cluster has been analyzed. From the view of pairs analysis, the conclusion has been discussed.
\end{abstract}

Keywords: Icosahedral quasicrystal, Molecular dynamics simulations, $\operatorname{EAM}($ embeded atom method) potential 